\title{
Enhancing statistical performance of data-driven controller tuning via $\mathcal{L}_{2}$-regularization
}

\author{
Simone Formentin, Alireza Karimi \\ Laboratoire d'Automatique, École Polytechnique Fédérale de Lausanne (EPFL), CH-1015 Lausanne (Switzerland) \\ e-mail: \{simone.formentin, alireza.karimi\}@epfl.ch
}

\begin{abstract}
Noniterative data-driven techniques are design methods that allow optimal feedback control laws to be derived from inputoutput (I/O) data only, without the need of a model of the process. A drawback of these methods is that, in their standard formulation, they are not statistically efficient. In this paper, it is shown that they can be reformulated as $\mathcal{L}_{2}$-regularized optimization problems, by keeping the same assumptions and features, such that their statistical performance can be enhanced using the same identification dataset. A convex optimization method is also introduced to find the regularization matrix. The proposed strategy is finally tested on a benchmark example in digital control system design.
\end{abstract}

Key words: Data-driven control, identification for control, VRFT, CbT, regularization

\section{INTRODUCTION}

In the standard approach to controller design, a model of the plant is identified from data or developed from first principles. In fixed-order model reference control, this model is then used to design a controller that minimizes the model reference criterion and a controller-order reduction step is carried out before implementation [1]. Various controller design approaches are available for the design of a high performance controller for the available model [2]. However, this controller is not necessarily optimal when connected to the plant, as the control performance is limited by modeling errors. Hence, several data-driven controller tuning techniques have been proposed to avoid the problem of undermodeling and to facilitate the design of fixed-order controllers, both iteratively e.g. [3], [4] and non-iteratively e.g. [5], [6]. In all these approaches, only the final control cost is taken into account and the controller is directly identified from data, without first deriving a model of the process. This paper will deal with the noniterative approaches, whereof the most interesting features are that the internal stability can be guaranteed [6] and, since the controller parameter estimation problem is convex, the global optimum can be found. Specifically, two of the most widespread techniques will be considered as examples of this approach, i.e. Virtual Reference Feedback Tuning (VRFT [5]) and noniterative Correlation-based Tuning (CbT [6]).
Various application examples have shown the effectiveness of the above methods in simulation and real-world systems, see e.g. [7], [8], [9], [10]. However, these methods suffer from the drawback that, being based on errors-in-variables estimation [11], they are not statistically efficient, i.e. the Cramér-Rao lower bound cannot be achieved in all the cases [12].

The aim of this paper is then two-fold. Firstly, it will be shown how the above data-driven design techniques can be reformulated as $\mathcal{L}_{2}$-regularized optimization problems. By doing so, the statistical performance of the methods will be significantly improved, as $\mathcal{L}_{2^{-}}$ regularization is very suited to cope with high variance estimation problems in system identification [13]. A very important observation is that the bias introduced by the penalty term used in the regularization procedure is not meaningful itself, as the aim of data-driven design is the maximization of the model-matching performance for any length of the data-set.

The interest in regularization has been recently renewed for linear system identification prompted by the new Bayesian perspective given in [14] and its follow-up (see, e.g. [15]). Specifically, the idea of the above papers is to see the identification of the impulse response of a system as an infinite-dimensional space learning problem, instead of considering finite-dimensional parameterization. In this framework, regularization techniques correspond to impose certain prior distributions on the impulse response parameters and the same philosophy will be applied in this work (for the first time) for con- 
troller identification.

The second goal of this paper is to provide a method to find the optimal kernel, or regularization matrix, for the regularized estimate, using a Bayesian perspective to employ the available preliminary knowledge on the system. Unlike the method proposed in [16], this procedure will not require assumptions on the spectra of input. A data-driven solution using the same dataset for identification is proposed to infer the "a-priori" information needed to derive the optimal kernel. Specifically, a high order FIR is identified and used to compute the noisy regressor and the a-priori covariance matrix of the controller parameters. In simulation, it will be shown that this approach is better than using a regularized FIR model to design a model-reference controller.

It should be said herein that the problem of improving statistical performance of noniterative data-driven approaches has been already dealt with in [17], where optimal input design has been proposed as a solution. Here, unlike [17], the improvement is obtained without additional experiments. Nevertheless, since the performance given by the method in this work is inputdependent, results in [17] could still be used to further improve the closed-loop model-matching, whenever new experiments are possible.

The outline of the paper is as follows. In Section 2, backgrounds on noniterative data-driven techniques are briefly recalled. In Section 3, regularized estimate for the above techniques will be introduced and analyzed, while a convex optimization method for kernel selection is formulated in Section 4. The regularized methods is compared with the standard ones on the benchmark example [18] in Section 5. Some concluding remarks end the paper.

\section{BACKGROUNDS}

Consider the unknown LTI SISO stable plant $G\left(q^{-1}\right)$, where $q^{-1}$ denotes the backward shift operator. The objective of the model-reference control problem is to design a linear, fixed-order controller $K\left(q^{-1}, \rho\right)$, parameterized through $\rho$, for which the closed-loop system matches a given stable strictly proper reference model $M\left(q^{-1}\right)$. More specifically, let the controller parameterization be

$$
K\left(q^{-1}, \rho\right)=\beta^{T}\left(q^{-1}\right) \rho,
$$

where $\beta\left(q^{-1}\right)$ is a vector of linear discrete-time basis functions

$$
\beta^{T}\left(q^{-1}\right)=\left[\beta_{1}\left(q^{-1}\right), \beta_{2}\left(q^{-1}\right), \ldots, \beta_{n}\left(q^{-1}\right)\right]
$$

Formally, the aim is to find the vector of parameters that minimizes the (filtered) $\mathcal{H}_{2}$-norm of the difference between the reference model and the achieved closedloop system:

$$
J_{m r}(\rho)=\left\|\left(\frac{G K(\rho)}{1+G K(\rho)}-M\right) W\right\|_{2}^{2},
$$

where $W\left(q^{-1}\right)$ is a user-defined frequency-weighting filter. The goal can be interpreted as to find the minimizer of the $\mathcal{L}_{2}$-norm of the matching error signal $\varepsilon$ in Fig. 1, when the reference signal is a white noise of unit variance and $v(t)=0, \forall t$. From now on, the minimizer of (1) will be referred to as "optimal controller" and will be denoted by $K_{o}\left(q^{-1}\right)=K\left(q^{-1}, \rho_{o}\right)=\beta^{T}\left(q^{-1}\right) \rho_{o}$.

Consider now that an open-loop collection of input-

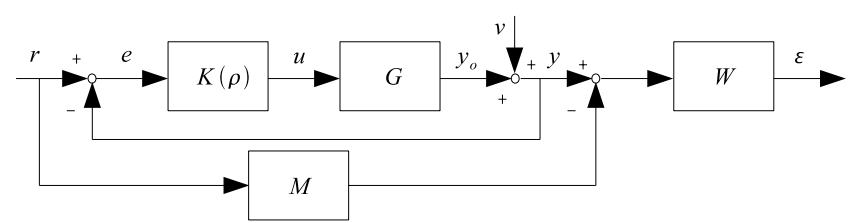

Fig. 1. Model reference control problem.

output (I/O) data $\{u(t), y(t)\}_{t=1, \ldots, N}$ is available and let the output $y(t)$ be affected by the additive noise

$$
v(t)=H\left(q^{-1}\right) d(t)
$$

where $H\left(q^{-1}\right)$ is an unknown stable LTI system and $d(t)$ is a zero mean white Gaussian noise with variance of $\sigma^{2}$, uncorrelated with $u(t)$.

In standard "indirect data-driven" or "model-based" approaches, the above objective can be achieved by identifying from data a model $\hat{G}$ of the plant and designing a model-based controller $K(\hat{G})$ as

$$
K(\hat{G})=\frac{M}{\hat{G}(1-M)} .
$$

A comparison between the direct data-driven and modelbased approach for model reference control problem is performed in [19].

\subsection{Noniterative correlation-based Tuning}

Let the ideal controller $K^{*}$ be defined as:

$$
K^{*}=\frac{M}{G(1-M)}
$$

Note that with the assumption that $M \neq 1$, this controller exists but may be non causal or of very high order. Based on this definition, the ideal sensitivity function can be seen as:

$$
\frac{1}{1+G K^{*}}=1-M
$$

and (1) can be rewritten as

$$
J_{m r}(\rho)=\left\|\frac{G K^{*}-G K(\rho)}{\left(1+G K(\rho)\left(1+G K^{*}\right)\right.} W\right\|_{2}^{2} .
$$


Approximating $(1+G K(\rho))^{-1}$ by $1-M$ leads to the following convex approximation of the model reference criterion:

$$
J(\rho)=\|(K(\rho)(1-M) G-M)(1-M) W\|_{2}^{2} .
$$

Consider now the scheme in Fig. 2, in the case where the signals are noiseless and $r(t)=u(t)$. In the noiseless

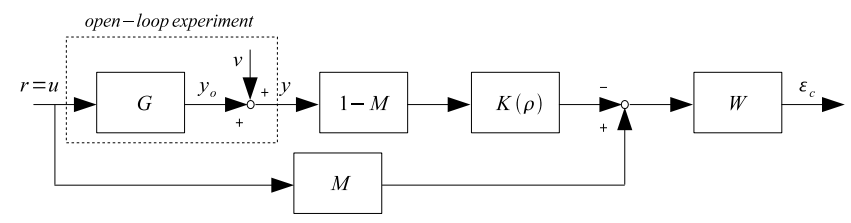

Fig. 2. Tuning scheme for Correlation-based Tuning.

setting, the model matching error $\varepsilon_{c}(t, \rho)$ defined in Fig.2 and its $\mathcal{L}_{2}$-norm can be directly computed from I/O data (unlike $\varepsilon(t, \rho))$ as

$$
\begin{aligned}
\varepsilon_{c}(t, \rho) & =W M r(t)-W(1-M) K(\rho) G r(t) \\
& =W M u(t)-W(1-M) K(\rho) y(t) .
\end{aligned}
$$

When data are collected in a noisy environment, the method resorts to the correlation approach to identify the controller. Specifically, an extended instrumental variable $\zeta(t)$ correlated with $u(t)$ and uncorrelated with $v(t)$ is introduced to decorrelate the error signal $\varepsilon_{c}(t)$ and $u(t) . \zeta(t)$ is defined as

$$
\zeta(t)=[u(t+l), \ldots, u(t), \ldots, u(t-l)]^{T},
$$

where $l$ is a sufficiently large integer. The correlation function and the correlation criterion are defined, respectively, as

$$
f_{N, l}(\rho)=\frac{1}{N} \sum_{t=1}^{N} \zeta_{L}(t) \varepsilon_{c}(t, \rho)
$$

where $\zeta_{L}(t)=L_{c}\left(q^{-1}\right) \zeta(t)$, and

$$
J_{N, l}(\rho)=f_{N, l}^{T}(\rho) f_{N, l}(\rho)
$$

In [6], it has been proven that

$$
\lim _{N, l \rightarrow \infty, l / N \rightarrow 0} J_{N, l}(\rho)=J(\rho) .
$$

if $L_{c}\left(q^{-1}\right)$ is chosen such that:

$$
L_{c}\left(e^{-j \omega}\right)=\frac{1-M\left(e^{-j \omega}\right)}{\Phi_{u}(\omega)}
$$

where $\Phi_{u}(\omega)$ denotes the spectral density of $u(t)$.

\subsection{Virtual Reference Feedback Tuning}

The idea of Virtual Reference Feedback tuning was first proposed in [20] with the name of Virtual Reference Direct Design $\left(V R D^{2}\right)$ and subsequently fixed and extended in [5], [21] and [22] respectively for LTI, nonlinear and LPV systems.

The main idea to minimize (1) without identifying $G\left(q^{-1}\right)$ is to build a "virtual" closed-loop system, where the input and output signals are equal to $u(t)$ and $y(t)$ and the closed-loop transfer function corresponds to $M\left(q^{-1}\right)$. From such loop, the so-called "virtual reference" $r_{v}(t)$ and "virtual error" $e_{v}(t)$ signals can be computed as $r_{v}(t)=M^{-1}\left(q^{-1}\right) y(t)$ and $e_{v}(t)=r_{v}(t)-y(t)$. The control design issue is then reduced to an identification problem, where the optimal controller is the one that generates $u(t)$ when fed by $e_{v}(t)$. The criterion to be minimized is then

$$
J_{v r}^{N}(\rho)=\frac{1}{N} \sum_{t=1}^{N}\left(u_{L}(t)-K\left(q^{-1}, \rho\right) e_{L}(t)\right)^{2},
$$

where $u_{L}(t)=L_{v r}\left(q^{-1}\right) u(t), e_{L}(t)=L_{v r}\left(q^{-1}\right) e_{v}(t)$ and $L_{v r}\left(q^{-1}\right)$ is a suitable prefilter such that (12) is equal to the second-order Taylor expansion of (1) in the neighborhood of the minimum point [5]. More specifically, the frequency response of $L_{v r}\left(q^{-1}\right)$ must be such that

$$
L_{v r}\left(e^{-j \omega}\right)=\frac{M\left(e^{-j \omega}\right)\left(1-M\left(e^{-j \omega}\right)\right) W\left(e^{-j \omega}\right)}{\Phi_{u}^{1 / 2}(\omega)},
$$

where $\Phi_{u}^{1 / 2}(\omega)$ denotes a spectral factor of $\Phi_{u}(\omega)$.

For the final limit estimate not to be biased, basic instrumental variables [23] are used to counteract the effect of noise. In this situation, the controller parameters are computed as

$$
\hat{\rho}_{N}=\operatorname{Sol}\left\{\frac{1}{N} \sum_{t=1}^{N} \varphi_{2}(t)\left(\varphi^{T}(t) \rho-u_{L}(t)\right)=0\right\}
$$

\section{EXPLOITING REGULARIZATION}

From now on, it will be assumed that the given controller class is flexible enough to achieve the user-defined reference model. In such a way, deterministic and stochastic errors, due respectively to under-parameterization of the controller structure and noise, will not be mixed and the analysis of the statistical performance affected by the output noise will be clearly delineated.

In their classical formulation, data-driven methods provide a consistent estimate of the optimal controller, using the instrumental variable (IV) techniques to cope 
with measurement noise. However, it is known that in IV techniques the variance of the parameter estimate is larger than the Cramér-Rao lower bound [12].

Since the final goal in practice is the minimization of (1) for a given $\mathrm{N}$ and not to find an unbiased controller estimate, $\mathcal{L}_{2}$-regularization can be used, analogously to [13], to improve the quality of the estimates by introducing an additional tuning-knob to balance bias and variance effects.

Specifically, denote with the regularization matrix $D \in$ $\mathcal{R}^{n \times n}$ the above tuning knob. Then, the regularized estimation formulas for the methods in Section 2 are as follows. For CbT, the new estimate is defined as

$$
\hat{\rho}_{N}=\arg \min _{\rho}\left[J_{N, l}(\rho)+\rho^{T} D \rho\right]
$$

that is

$$
\hat{\rho}_{N}=\left(Q^{T} Q+D\right)^{-1} Q^{T} z,
$$

where

$$
\begin{aligned}
Q & =\frac{1}{N} \sum_{t=1}^{N} \zeta_{L}(t) \phi^{T}(t), z=\frac{1}{N} \sum_{t=1}^{N} \zeta_{L}(t) y_{d}(t), \\
\phi(t) & =\beta(1-M) y(t) \\
& =\beta(1-M) G u(t)+\beta(1-M) v(t) \\
& =\phi_{o}(t)+\tilde{\phi}(t),
\end{aligned}
$$

and $y_{d}(t)=\phi_{o}^{T}(t) \rho_{o}$, as $M$ is assumed to be achievable with the given controller parameterization.

Analogously, for VRFT with basic instrumental variables, the new controller identification problem is the following modification of (14)

$$
\hat{\rho}_{N}=\operatorname{Sol}\left\{\frac{1}{N} \sum_{t=1}^{N} \varphi_{2}(t)\left(\varphi^{T}(t) \rho-u_{L}(t)\right)+D \rho=0\right\},
$$

that is

$$
\begin{gathered}
\hat{\rho}_{N}=(S+D)^{-1} s \\
S=\frac{1}{N} \sum_{t=1}^{N} \varphi_{2}(t) \varphi^{T}(t), s=\frac{1}{N} \sum_{t=1}^{N} \varphi_{2}(t) u_{L}(t),
\end{gathered}
$$

where

$$
\begin{aligned}
\varphi(t) & =\beta\left(M^{-1}-1\right) L y(t) \\
& =\beta\left(M^{-1}-1\right) L G u(t)+\beta\left(M^{-1}-1\right) L v(t) \\
& =\varphi_{o}(t)+\tilde{\varphi}(t), \\
\varphi_{2}(t) & =\beta\left(M^{-1}-1\right) L y_{2}(t),
\end{aligned}
$$

$y_{2}(t)$ is the output of a second experiment on the plant $G$ and $u(t)=\varphi_{o}^{T}(t) \rho_{o}$, as $M$ is assumed to be achievable with the given controller parameterization. According to the theory in [5], the second experiment must be performed using the same input sequence $u(t)$ as the first one, such that the only difference between $y(t)$ and $y_{2}(t)$ is that the latter is characterized by a different realization of the noise $v(t)$.

Notice that (16) and (20) generally provide an estimate of $K_{o}$, with an additional bias due to $D$. Consider now the second order Taylor expansion of (1) around the optimum $\rho_{o}$

$$
\begin{aligned}
J_{m r}\left(\hat{\rho}_{N}\right)=\left.\left(\hat{\rho}_{N}-\rho_{o}\right)^{T} \frac{\partial^{2} J_{m r}}{\partial \rho^{2}}\right|_{\rho_{o}} & \left(\hat{\rho}_{N}-\rho_{o}\right) \\
& +\mathcal{O}_{2}\left(\hat{\rho}_{N}-\rho_{o}\right),
\end{aligned}
$$

where $\mathcal{O}_{2}\left(\hat{\rho}_{N}-\rho_{o}\right)$ collects all the terms of order higher than 2 . It should be noted that $J_{m r}(\rho)$ shares the same minimum and the same Hessian of (6) if $M$ is achievable, i.e., $K^{*} \in\{K(\rho)\}$ or $K^{*}=K\left(\rho_{o}\right)$. As a matter of fact, with this assumption the Hessian of $J$ in $\rho_{o}$ is

$$
\begin{aligned}
\Delta & =\left.\frac{\partial^{2} J}{\partial \rho^{2}}\right|_{\rho_{o}}=\frac{1}{\pi} \int_{-\pi}^{\pi} \frac{|W|^{2}|1-M|^{2}|M|^{2}}{\left|K\left(\rho_{o}\right)\right|^{2}} \beta \beta^{T} d \omega \\
& =\frac{1}{\pi} \int_{-\pi}^{\pi}|W|^{2}|1-M|^{4}|G|^{2} \beta \beta^{T} d \omega
\end{aligned}
$$

which is equal to the Hessian of $J_{m r}$

$$
\left.\frac{\partial^{2} J_{m r}}{\partial \rho^{2}}\right|_{\rho_{o}}=\frac{1}{\pi} \int_{-\pi}^{\pi} \frac{|W|^{2}|1-M|^{2}|G|^{2}}{\left|1+G K\left(\rho_{o}\right)\right|^{2}} \beta \beta^{T} d \omega .
$$

Therefore, (24) is well approximated by the second order Taylor expansion of (6), that is

$$
J\left(\hat{\rho}_{N}\right)=\left(\hat{\rho}_{N}-\rho_{o}\right)^{T} \Delta\left(\hat{\rho}_{N}-\rho_{o}\right),
$$

Since $\hat{\rho}_{N}$ depends on $D$ (see again (16) for CbT and (20) for VRFT), the kernel can then be used to minimize the total effect of bias and variance on the finite-sample error $J_{m r}\left(\hat{\rho}_{N}\right)$ for given $N$. Theoretically, the optimal $D \geq 0$ for assigned $N$ could be defined as

$$
\hat{D}=\arg \min _{D}\left[\left(\hat{\rho}_{N}(D)-\rho_{o}\right)^{T} \Delta\left(\hat{\rho}_{N}(D)-\rho_{o}\right)\right],
$$

where $\hat{\rho}_{N}$ is as in (16) or in (20) and the dependence of $\hat{\rho}_{N}$ on $D$ has been highlighted. However, since in (28) the best $D$ fundamentally depends on the optimal parameter vector $\rho_{o}$, the aforementioned optimization problem cannot be solved in practice.

A realistic strategy is instead to define the optimal $D \geq 0$ according to a Bayesian perspective, that is, as

$$
\hat{D}=\arg \min _{D} \mathbb{E}_{\rho_{o} \in \Theta_{o}}\left[\left(\hat{\rho}_{N}(D)-\rho_{o}\right)^{T} \Delta\left(\hat{\rho}_{N}(D)-\rho_{o}\right)\right],
$$

where $\rho_{o}$ is no longer a set of deterministic parameters, but a random vector. The best $D$ is then now computed 
inside a set of candidate optimal controllers $\Theta_{o}$. Specifically, still following the Bayesian approach, let assume in this work that the prior distribution of $\rho_{o}$ is Gaussian where $\rho_{o} \sim \mathcal{N}(0, \Xi)$, and that the "a-priori" covariance $\Xi=\mathbb{E}_{\rho_{o} \in \Theta_{o}}\left[\rho_{o} \rho_{o}^{T}\right]$ is given. Problem (29) will be discussed in detail in the next Section, where a solution based on convex optimization will be proposed.

Notice that such a regularization term is dependent on the realization of the output noise $v$, as the only prior information available for kernel selection is the set of open-loop data. However, the so-found $\hat{D}$ will be such that the estimate is largely improved.

\section{KERNEL SELECTION}

Notice that the optimization problem (29) is nonlinear in $D$. In [13] and [16], it has been shown that an analogous problem in system identification (i.e. to find the kernel minimizing the mean square error) can be solved analytically if the input sequence and the disturbances are white noises. In the data-driven control framework, both the input and the disturbance of the system to identify, i.e. the controller, are generally not white, even if $u(t)$ and $v(t)$ are white (see again Fig. 2 for CbT and the expression of the virtual error $e_{v}$ in Section 2 for VRFT). The main results of this work concerning optimal kernel selection are the two theorems presented next. To clearly introduce the statements, first define the noisy part of the regressors

$$
\begin{aligned}
& F=\frac{1}{N} \sum_{t=1}^{N} \zeta_{L}(t) \tilde{\phi}^{T}(t) . \\
& R=\frac{1}{N} \sum_{t=1}^{N} \varphi_{2}(t) \tilde{\varphi}^{T}(t) .
\end{aligned}
$$

for $\mathrm{CbT}$ and VRFT, respectively. Moreover, let $\Gamma$ be a Cholesky factor [24] of $\Xi$, i.e. a triangular matrix such that $\Xi=\Gamma \Gamma^{T}$. From [5] and [6] it is known that, when data are noiseless and prefilters $L_{c}$ and $L_{v r}$ are used,

$$
\left.\lim _{N \rightarrow \infty} \frac{\partial^{2} J_{N, l}(\rho)}{\partial \rho^{2}}\right|_{\rho_{o}}=\left.\lim _{N \rightarrow \infty} \frac{\partial^{2} J_{v r}^{N}(\rho)}{\partial \rho^{2}}\right|_{\rho_{o}}=\Delta
$$

such that $\Delta$ can be computed without relying on the (unknown) controller parameters $\rho_{o}$. As a matter of fact, not only the data-driven cost criteria do not depend on $G$, but, since they are quadratic functions of $\rho$, their second order derivatives in the optimum do not depend on $\rho_{o}$ either. Equation (32) will be used later on to compute $\Delta$. The two theorems are as follows.

Theorem 1 Set $D^{(0)}$ as a preliminary estimate of $D$ and consider the iterative convex optimization problem

$$
\hat{D}_{c b}^{(i+1)}=\arg \min _{D} \gamma
$$

$$
\begin{gathered}
\text { s.t. } \gamma-\operatorname{tr}\left\{\Omega_{c b}\right\}>0, \\
{\left[\begin{array}{cc}
\Omega_{c b} & \Gamma^{T}\left(D^{T}+F^{T} Q\right) \\
\left(Q^{T} F+D\right) \Gamma & Z_{c b}
\end{array}\right]>0,} \\
\left(Q Q^{T}+D^{(i) T}\right) \Delta^{-1}\left(Q^{T} Q+D^{(i)}\right)+Q Q^{T} \Delta^{-1}\left(D-D^{(i)}\right) \\
+\Delta^{-1} Q^{T} Q\left(D-D^{(i)}\right)-Z_{c b}>0, \\
D \geq 0,
\end{gathered}
$$

where $Z_{c b} \in \mathcal{R}^{n \times n}$ and $\Omega_{c b} \in \mathcal{R}^{n \times n}$ are slack matrices. The solution of the above iterative algorithm converges to a local minimum of the nonlinear optimization problem in (29), where $\hat{\rho}_{N}$ is computed via (16).

Proof 1 See the Appendix.

Theorem 2 Set $D^{(0)}$ as a preliminary estimate of $D$ and consider the iterative convex optimization problem

$$
\begin{gathered}
\hat{D}_{v r}^{(i+1)}=\arg \min _{D} \gamma \\
\text { s.t. } \gamma-\operatorname{tr}\left\{\Omega_{v r}\right\}>0, \\
{\left[\begin{array}{cc}
\Omega_{v r} & \Gamma^{T}\left(D^{T}+R^{T}\right) \\
(R+D) \Gamma & Z_{v r}
\end{array}\right]>0,} \\
\left(S^{T}+D^{(i) T}\right) \Delta^{-1}\left(S+D^{(i)}\right)+S^{T} \Delta^{-1}\left(D-D^{(i)}\right) \\
+\Delta^{-1} S\left(D-D^{(i)}\right)-Z_{v r}>0, \\
D \geq 0,
\end{gathered}
$$

where $Z_{v r} \in \mathcal{R}^{n \times n}$ and $\Omega_{v r} \in \mathcal{R}^{n \times n}$ are slack matrices. The solution of the above iterative algorithm converges to a local minimum of the nonlinear optimization problem in (29), where $\hat{\rho}_{N}$ is computed via (20).

Proof 2 See the Appendix.

Like any regularization procedure, also the given algorithm with the previous results can be interpreted as a way to introduce some "a-priori" information inside the optimization problem. This is done three times: while using $\Xi$, when computing the noisy regressors and when the Hessian of the cost function is numerically approximated as in (32).

Firstly, $\Xi$ is used here because, according to the Bayesian point of view, $\rho_{o}$ can be seen as a random vector whose "a-priori" density is $\mathcal{N}(0, \Xi)$ and "a-posteriori" density is $\mathcal{N}\left(\hat{\rho}_{N},\left(\Xi^{-1}+P^{-1}\right)^{-1}\right)$. Specifically, for $\mathrm{CbT}, \hat{\rho}_{N}$ is given by (16) and $P$ is the variance of the $\mathrm{CbT}$ estimate [12]. Analogously, for VRFT, $\hat{\rho}_{N}$ is given by (20) and $P$ is the variance of the VRFT estimate [12].

Secondly, preliminary information on the controller is 
used when computing $F$ and $R$, because they depend on the noise regressors $\tilde{\phi}(t)$ and $\tilde{\varphi}(t)$. These can be computed either if the time history of the noise signal can be reconstructed or if a preliminary ("a-priori") estimate of $\hat{\rho}_{N}$ is given (such that an estimate of $\phi_{o}(t)$ and $\varphi_{o}(t)$ can be assessed and $\left.\tilde{\phi}(t)=\phi(t)-\phi_{o}(t), \tilde{\varphi}(t)=\varphi(t)-\varphi_{o}(t)\right)$. Finally, the estimate of the noiseless regressors can also be employed for computing $\Delta$ as indicated in (32).

In practical situations, the needed preliminary knowledge about the optimal controller could be derived in different ways. In this paper, the following philosophy is employed:

1) a high-order FIR model $\hat{G}$ is identified from I/O data (notice that it is another convex procedure via $\mathrm{PEM}$ ) and an estimate of the noiseless output is computed as $\hat{y}=\hat{G} u$;

2) CbT or VRFT formula, respectively (16) and (20), can be used to find a preliminary estimate $\tilde{\rho}$ of the controller parameters using $u$ and $\hat{y}$; moreover, from $\hat{y}$, both $F, R$ and the approximated hessian in (32) can also be derived;

3) the kernel $\Xi$ for the optimization of the regularization matrix $D_{N}$ can then be computed as $\tilde{\rho} \tilde{\rho}^{T}$.

One could object that this procedure to find a suited "a-priori" information about the controller makes the method no longer data-driven but "model-based", as $\hat{G}$ is used. This is not completely true, because the model is not directly used to compute the controller but it is only employed to find the best penalty term in the regularized cost. In the next section, it will be shown how this fact is crucial to determine the closed-loop performance and how the regularized controller identification leads to better performance than model-based design even where a regularized FIR model is employed. Notice also that the whole controller identification procedure is convex (whereas regularized FIR identification is not) and does not require to select a model structure for $\hat{G}$.

Remark. The difference between standard and regularized estimate becomes more evident as the signal-to-noise ratio (SNR) decreases. At limit, for noiseless data, the best regularized estimate is equal to the standard one, i.e. $D_{N}=0$, as the estimate is unbiased and the variance is zero. For noisy data of large variance, regularization provides an additional bias but with better overall modelmatching performance, as illustrated in the next section.

\section{SIMULATION EXAMPLE}

Consider the flexible transmission system introduced as a benchmark for digital control design in [18]. The plant is described by the discrete-time model

$$
G\left(q^{-1}\right)=\frac{0.28261 q^{-3}+0.50666 q^{-4}}{A\left(q^{-1}\right)}
$$

where $A\left(q^{-1}\right)=1-1.41833 q^{-1}+1.58939 q^{-2}-$ $1.31608 q^{-3}+0.88642 q^{-4}$. Let a pseudo-random-binarysignal (PRBS) of number of samples $N=1000$ be used to feed the system. Let also the measurement be white and such that the signal-to-noise ratio is 5 , that is, let $H\left(q^{-1}\right)=1$ and the variance of $v$ be $\operatorname{var}\left[y_{o}(t)\right] / 5^{2}$, where $\operatorname{var}\left[y_{o}(t)\right]$ is the variance of the noiseless output $y_{o}(t)$. Finally, the frequency-weighting function $W\left(q^{-1}\right)=1$, the set of available controllers is

$$
K(\rho)=\frac{\rho_{0}+\rho_{1} q^{-1}+\rho_{2} q^{-2}+\rho_{3} q^{-3}+\rho_{4} q^{-4}+\rho_{5} q^{-5}}{1-q^{-1}}
$$

and the control objective is defined as a reference model that allows the perfect matching to be achieved, i.e.

$$
M\left(q^{-1}\right)=\frac{G\left(q^{-1}\right) K\left(q^{-1}, \rho_{o}\right)}{1+G\left(q^{-1}\right) K\left(q^{-1}, \rho_{o}\right)},
$$

where the optimal controller is in the controller set and its parameters are

$\rho_{o}=[0.2045,-0.2715,0.2931,-0.2396,0.1643,0.0084]^{T}$.

A Monte-Carlo simulation with 100 running experiments is performed, using a different noise realization for each experiment, and the corresponding $\mathrm{CbT}$ and VRFT controllers are computed. For CbT, the length of the instrumental variable vector is $l=35$, which corresponds to the approximate length of the impulse response of $M$. Concerning VRFT, since it requires two sets of data for building the instrumental variable, each experiment is made of 500 samples for a fair comparison between the methods. To assess the final closed-loop performance, an estimate of $\mathbb{E}\left[J_{m r}\left(\hat{\rho}_{N}\right)\right]$ in the minimum $\hat{\rho}_{N}$ is computed by sample mean. The same test has been done for the regularized estimation method presented in this work, where $D$ is found via the procedure in Section 4 using only one iteration after the trivial initialization $D_{0}=0$. The model used to devise the priors for the control design task is a FIR of order 200, that is flexible enough to accurately fit the impulse response of (43).

The final model-matching results are illustrated in Table I, where standard estimation, regularized estimation with model-based kernel and ideal regularized estimation (i.e. regularized estimation with the kernel using $\left.\rho_{o}\right)$ are compared with FIR model-based control design. As an example, Fig. 3 and 4 show the accuracy of the magnitude Bode plots and the maximum improvement achievable with regularization.

The main findings for this example from the above tests can be summarized as follow:

- the performance achievable by ideal regularization is always better than standard data-driven tuning;

- the achievable regularized estimate (i.e., the one with the MB kernel) is far from the ideal one, however it enhances in any case the statistical performance of the methods: 
- in case of CbT, the achievable estimate is only slightly better than the standard method, however, at this stage, the result is important as this solution represents the best achievable statistical performance in the CbT design framework among the existing ones;

- it is better to use the FIR model of the system to provide the kernel of the regularized controller estimation than using it to design a model-based controller; notice also that by using regularization directly in control design, the whole procedure is convex, whereas the marginal likelihood approach in [16] is not;

- the method proves very effective when the variance is large, i.e. in VRFT estimate where basic instrumental variable are used (not only the mean cost decreases, but also all the controllers given by the regularized estimate are stabilizing, without adding any additional constraint). Notice that using the standard VRFT method, 5/100 controllers turn out to be destabilizing. In these cases, it is always possible to add the stability constraint introduced in [6], but the results would become more conservative;

- from a user perspective, the best performance among all the solutions is given by the regularized CbT method.

\section{CONCLUSIONS}

In this paper, the noniterative direct techniques introduced in [5] and [6] have been reformulated as $\mathcal{L}_{2}$-regularized optimization problems, where the biasvariance trade-off can be suitably optimized. In the paper, the regularization matrix has been fully parameterized and a convex optimization problem has been proposed for its design. Results on the benchmark example proposed in [18] show that the statistical performance is significantly improved in both the methods. Future work will focus on data-driven estimation of the prior information without using FIR modeling.

\section{References}

[1] B. Anderson and Y. Liu, "Controller reduction: concepts and approaches," Automatic Control, IEEE Transactions on, vol. 34, no. 8, pp. 802-812, 1989.

[2] J. Doyle, B. Francis, and A. Tannenbaum, Feedback control theory. Macmillan New York, 1992, vol. 134.

[3] H. Hjalmarsson, "Iterative feedback tuning - an overview," Int J. Adapt. Control Signal Process., 16, 2002.

[4] A. Karimi, L. Miskovic, and D. Bonvin, "Iterative correlationbased controller tuning," International Journal of Adaptive Control and Signal Processing, vol. 18, no. 8, pp. 645-664, 2004.

[5] M. Campi, A. Lecchini, and S. Savaresi, "Virtual reference feedback tuning: a direct method for the design of feedback controllers," Automatica, vol. 38, no. 8, pp. 1337-1346, 2002.

[6] K. van Heusden, A. Karimi, and D. Bonvin, "Data-driven model reference control with asymptotically guaranteed stability," International Journal of Adaptive Control and Signal Processing, vol. 25, no. 4, pp. 331-351, 2011.

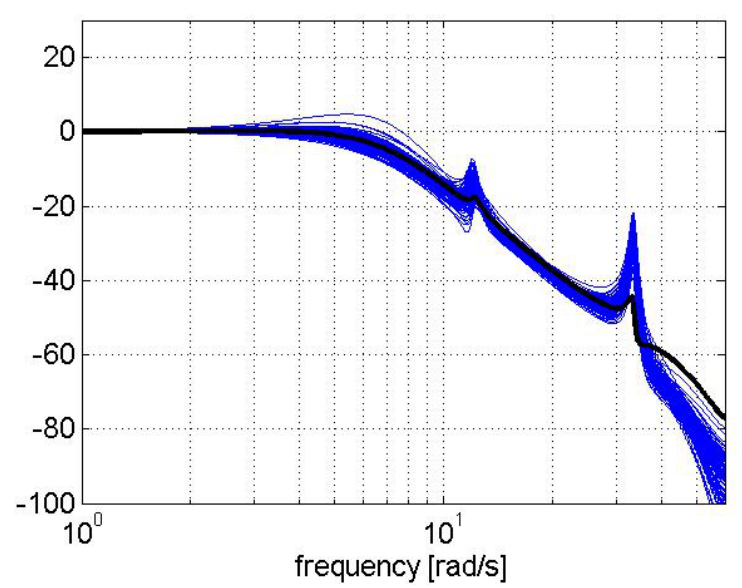

(a)

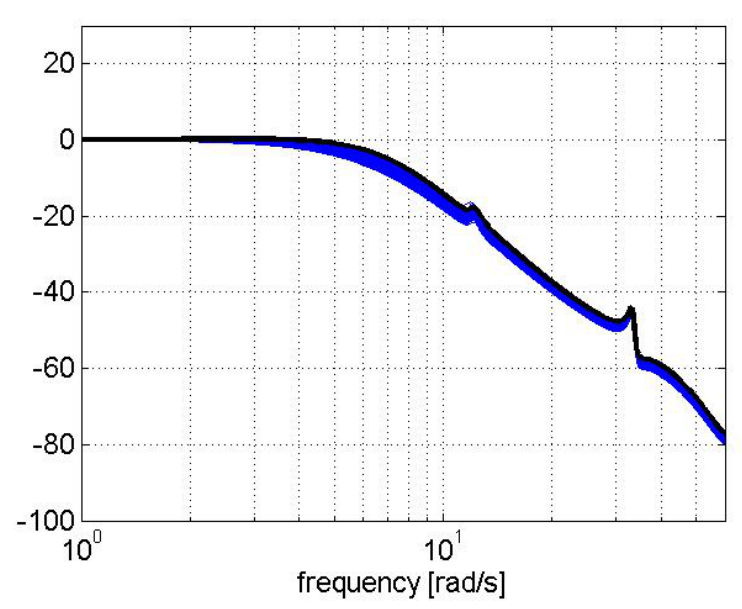

(b)

Fig. 3. Magnitude Bode plots: $M$ (black solid line), achieved closed-loop performance with standard CbT design (blue lines, above), achieved closed-loop performance with regularized CbT design (blue lines, below).

[7] S. Formentin, P. De Filippi, M. Corno, M. Tanelli, and S. Savaresi, "Data-driven design of braking control systems," IEEE Transactions on Control Systems Technology, vol. 21, no. 1, pp. 1-8, 2012.

[8] J. Rojas, F. Tadeo, and R. Vilanova, "Control of a PH neutralization plant using the VRFT framework," in IEEE International Conference on Control Applications, pp. 926931, Yokohama, September 8-10, 2010.

[9] K. Van Heusden, A. Karimi, D. Bonvin, A. Den Hamer, and M. Steinbuch, "Non-iterative data-driven controller tuning with guaranteed stability: Application to direct-drive pickand-place robot," in IEEE International Conference on Control Applications, pp. 1005-1010, Yokohama, September 8-10, 2010.

[10] S. Formentin, M. Corno, S. Savaresi, and L. Del Re, "Direct data-driven control of linear time-delay systems," Asian Journal of Control, vol. 14, no. 3, pp. 652-663, 2012.

[11] T. Soderstrom, "Errors-in-variables methods in system identification," Automatica, vol. 43, no. 6, pp. 939-958, 2007. 
Table 1

Mean values (100 experiments) of the achieved performance $J_{m r}$ (normalized with respect to the model-based case) using PRBS input and white noise disturbance.

\begin{tabular}{|c|c|c|c|c|c|c|}
\hline Model-based (MB) & CbT & rCbT - MB kernel & ideal rCbT & VRFT & rVRFT - MB kernel & ideal rVRFT \\
\hline \hline 1 & 0.557 & 0.407 & 0.253 & 1.420 & 0.981 & 0.272 \\
\hline
\end{tabular}

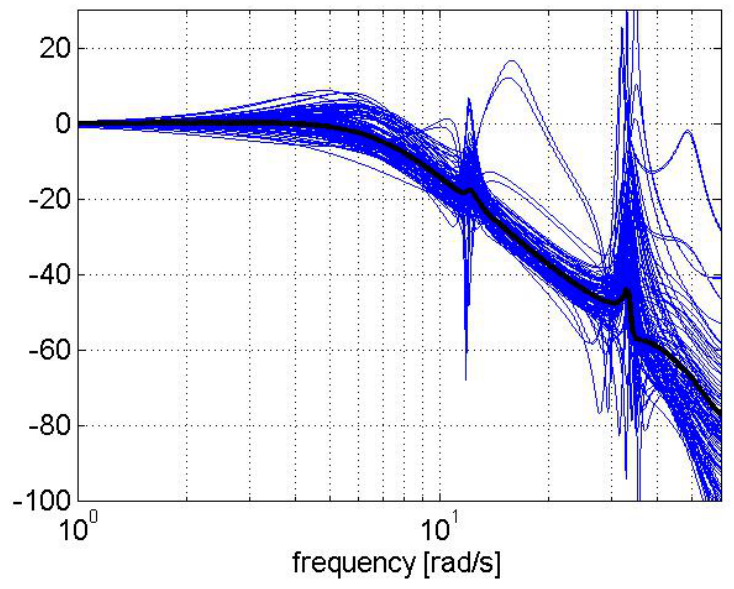

(a)

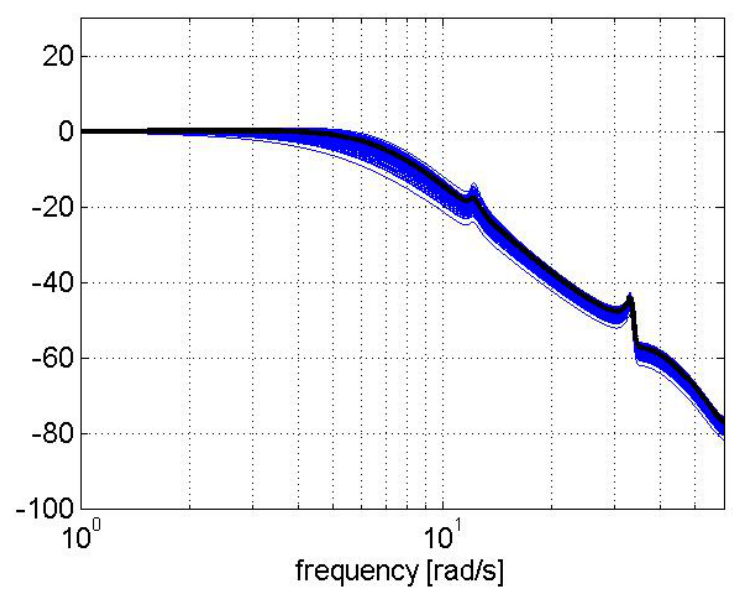

(b)

Fig. 4. Magnitude Bode plots: $M$ (black solid line), achieved closed-loop performance with standard VRFT design (blue lines, above), achieved closed-loop performance with regularized VRFT design (blue lines, below).

[12] K. van Heusden, A. Karimi, and T. Söderström, "On identification methods for direct data-driven controller tuning," International Journal of Adaptive Control and Signal Processing, vol. 25, no. 5, pp. 448-465, 2011.

[13] T. Chen, H. Ohlsson, and L. Ljung, "On the estimation of transfer functions, regularizations and gaussian processesrevisited," in Automatica, 2012.

[14] G. Pillonetto and G. De Nicolao, "A new kernel-based approach for linear system identification," Automatica, vol. 46, no. 1, pp. 81-93, 2010.

[15] G. Pillonetto, A. Chiuso, and G. De Nicolao, "Prediction error identification of linear systems: A nonparametric gaussian regression approach," Automatica, vol. 47, no. 2, pp. 291-305, 2011.

[16] T. Chen, H. Ohlsson, G. Goodwin, and L. Ljung, "Kernel selection in linear system identification part ii: A classical perspective," in 50th IEEE Conference on Decision and Control and European Control Conference (CDC-ECC), 2011.

[17] S. Formentin, A. Karimi, and S. Savaresi, "Optimal input design for direct data-driven tuning of model-reference controllers," Automatica, vol. 49, no. 6, pp. 1874-1882, 2013.

[18] I. Landau, D. Rey, A. Karimi, A. Voda, and A. Franco, "A flexible transmission system as a benchmark for robust digital control," European Journal of Control, vol. 1, no. 2, pp. 77-96, 1995.

[19] S. Formentin, K. Heusden, and A. Karimi, "A comparison of model-based and data-driven controller tuning," International Journal of Adaptive Control and Signal Processing, DOI: 10.1002/acs.2415, 2013.

[20] G. Guardabassi and S. Savaresi, "Approximate feedback linearization of discrete-time non-linear systems using virtual input direct design," Systems \& Control Letters, vol. 32, no. 2, pp. 63-74, 1997.

[21] M. Campi and S. Savaresi, "Direct nonlinear control design: The virtual reference feedback tuning (vrft) approach," IEEE Transactions on Automatic Control, vol. 51, no. 1, pp. 14-27, 2006.

[22] S. Formentin and S. Savaresi, "Virtual Reference Feedback Tuning for linear parameter-varying systems," in IFAC World Congress, Milan, 2011.

[23] L. Ljung, System identification: theory for the user. PrenticeHall Englewood Cliffs, NJ, 1987.

[24] S. Boyd and L. Vandenberghe, Convex optimization. Cambridge University Press, 2004.

[25] A. L. Yuille and A. Rangarajan, "The concave-convex procedure," Neural Computation, vol. 15, no. 4, pp. 915-936, 2003.

\section{APPENDIX}

In the proofs, the symbol $\mathbb{E}_{\rho_{o} \in \Theta_{o}}$ will be replaced by $\mathbb{E}$ for the sake of space.

Proof of Theorem 1 : To start with, consider a single iteration. Take (16) and rewrite $z$ as

$$
\begin{aligned}
z & =\frac{1}{N} \sum_{t=1}^{N} \zeta_{L}(t) \phi_{o}^{T}(t) \rho_{o} \\
& =\frac{1}{N} \sum_{t=1}^{N} \zeta_{L}(t) \phi^{T}(t) \rho_{o}-\frac{1}{N} \sum_{t=1}^{N} \zeta_{L}(t) \tilde{\phi}^{T}(t) \rho_{o} \\
& =Q \rho_{o}-F \rho_{o}
\end{aligned}
$$


with obvious definition for $F$. The estimate (16) then becomes:

$$
\begin{aligned}
\hat{\rho}_{N} & =\left(Q^{T} Q+D\right)^{-1} Q^{T} Q \rho_{o}-\left(Q^{T} Q+D\right)^{-1} Q^{T} F \rho_{o} \\
& =\rho_{o}-\left(Q^{T} Q+D\right)^{-1} Q^{T}(D+F) \rho_{o} .
\end{aligned}
$$

Notice that $\rho_{o}-\hat{\rho}_{N}$ is not zero for the presence of two additional random terms. The first term

$$
\hat{\rho}_{b 1, N}=\left(Q^{T} Q+D\right)^{-1} D \rho_{o}
$$

is zero if $D=0$, while the second term

$$
\hat{\rho}_{b 2, N}=\left(Q^{T} Q+D\right)^{-1} Q^{T} F \rho_{o}
$$

is zero only if data are noiseless or as $N \rightarrow \infty$. By defining $X_{c b}=Q^{T} Q+D$, Problem (29) is reformulated as

$$
\begin{gathered}
\hat{D}_{c b}=\arg \min _{D} \gamma, \\
\text { s.t. } D \geq 0, \text { and } V(D)<\gamma,
\end{gathered}
$$

where $V(D)$ is defined as

$V(D)=\mathbb{E}\left[\rho_{o}^{T}\left(D^{T}+F^{T} Q\right) X_{c b}^{-1} \Delta X_{c b}^{-1}\left(Q^{T} F+D\right) \rho_{o}\right]$.

The above optimization problem is nonlinear and $\rho_{o^{-}}$ dependent. However, since $V(D)$ is a scalar, it can be rewritten as

$$
\operatorname{tr}\left\{\mathbb{E}\left[\rho_{o}^{T}\left(D^{T}+F^{T} Q\right) X_{c b}^{-1} \Delta X_{c b}^{-1}\left(Q^{T} F+D\right) \rho_{o}\right]\right\},
$$

or, using the cyclic property of the trace, as

$$
\begin{aligned}
& \operatorname{tr}\left\{\mathbb{E}\left[\rho_{o}^{T} \rho_{o}\right]\left(D^{T}+F^{T} Q\right) X_{c b}^{-1} \Delta X_{c b}^{-1}\left(Q^{T} F+D\right)\right\}= \\
& \quad \operatorname{tr}\left\{\Xi\left(D^{T}+F^{T} Q\right) X_{c b}^{-1} \Delta X_{c b}^{-1}\left(Q^{T} F+D\right)\right\} .
\end{aligned}
$$

Now, since $\Xi$ is known and definite positive, a Cholesky factor, i.e. a matrix $\Gamma$ such that $\Xi=\Gamma \Gamma^{T}[24]$, can be easily computed and $V(D)$ can finally be written as

$$
V(D)=\operatorname{tr}\left\{\Gamma^{T}\left(D^{T}+F^{T} Q\right) X_{c b}^{-1} \Delta X_{c b}^{-1}\left(Q^{T} F+D\right) \Gamma\right\}
$$

The constraint $V(D)<\gamma$ now depends only on the "apriori" variance of $\rho_{o}$, that is supposed known. Then, using the Schur Lemma [24], $V(D)<\gamma$ is equivalent to $\operatorname{tr}\left\{\Omega_{c b}\right\}<\gamma$ where $\Omega_{c b} \in \mathcal{R}^{n \times n}$ is a slack variable such that

$$
\left[\begin{array}{cc}
\Omega_{c b} & \Gamma^{T}\left(D^{T}+F^{T} Q\right) \\
\left(Q^{T} F+D\right) \Gamma & X_{c b} \Delta^{-1} X_{c b}
\end{array}\right]>0,
$$

Now, define another slack variable $Z_{c b} \in \mathcal{R}^{n \times n}$ such that

$$
Z_{c b}<X_{c b} \Delta^{-1} X_{c b}^{T}
$$

and replace $X_{c b} \Delta^{-1} X_{c b}^{T}$ with it to make (54) convex. By doing this, the concave constraint in (55) appears. However, such a constraint can be replaced by an inner convex approximation, by rewriting the first order expansion of $X_{c b} \Delta^{-1} X_{c b}^{T}$ with respect to $D$ around the given previous estimate $D^{(i)}$, thus obtaining (36). Starting from $D^{(0)}$ and updating each estimate by solving the above convex optimization problem, the overall iterative algorithm can be seen as a so-called "convex-concave" procedure. As proven in [25], such a procedure converges to a local minimum of the original problem, that is, the problem in (29).

Proof of Theorem 2: This proof follows the same line of the previous one. Consider the single iteration first. Take (20) and rewrite $s$ as

$$
\begin{aligned}
s & =\frac{1}{N} \sum_{t=1}^{N} \varphi_{2}(t) \varphi_{o}(t) \rho_{o} \\
& =\frac{1}{N} \sum_{t=1}^{N} \varphi_{2}(t) \varphi^{T}(t) \rho_{o}-\frac{1}{N} \sum_{t=1}^{N} \varphi_{2}(t) \tilde{\varphi}^{T}(t) \rho_{o} \\
& =S \rho_{o}-R \rho_{o}
\end{aligned}
$$

with obvious definition for $R$. The estimate (20) then becomes

$$
\hat{\rho}_{N}=\rho_{o}-(S+D)^{-1}(D+R) \rho_{o}
$$

and, defining now $X_{v r}=S+D$, Problem (29) can be rewritten, for VRFT, as

$$
\begin{gathered}
\hat{D}_{v r}=\arg \min _{D} \gamma, \\
\text { s.t. } D \geq 0, \quad \text { and } \Psi(D)<\gamma,
\end{gathered}
$$

where

$$
\Psi(D)=\mathbb{E}\left[\rho_{o}^{T}\left(D^{T}+R^{T}\right) X_{v r}^{-1} \Delta X_{v r}^{-1}(R+D) \rho_{o}\right] .
$$

Now, by considering the same Cholesky factor $\Gamma$ of the definite positive matrix $\Xi$ and following the same rationale of the previous proof, it can be proved that (6) can be reformulated as

$$
\Psi(D)=\operatorname{tr}\left\{\Gamma^{T}\left(D^{T}+R^{T}\right) X_{v r}^{-1} \Delta X_{v r}^{-1}(R+D) \Gamma\right\} .
$$

Denoting the argument of the trace with the slack matrix $\Omega_{v r}$, the Schur Lemma and another slack matrix $Z_{v r}<$ $X_{v r} \Delta^{-1} X_{v r}^{T}$ can be used to introduce an inner convex approximation of the original problem, analogously to the $\mathrm{CbT}$ case. The overall algorithm is a concave-convex procedure and therefore it converges to a local minimum of the original problem in (29). 